\title{
Pulse-duration dependence of the isotopic effect in simple molecular ions driven by strong laser fields
}

\author{
G. Castiglia, P. P. Corso, E. Fiordilino, and F. Persico \\ Dipartimento di Fisica, Università degli studi di Palermo, Via Archirafi 36, I-90123 Palermo, Italy
}

(Received 29 November 2010; published 23 May 2011)

\begin{abstract}
In this paper we discuss isotopic effects in simple molecular ions subjected to strong laser fields. We show that the intensity of the emitted spectra strongly depends upon both the nuclear mass of the molecular ions and the laser pulse duration. In particular, for short pulse duration [up to 8 optical cycles (o.c.)], we confirm the trend described in the most studied case in which the high-order harmonic generation is more efficient for heavier isotopes; in contrast, an interesting physical phenomenon is predicted for pulses longer than 16 o.c. characterized by an inverse effect in which lighter molecular species are responsible for higher-order harmonic emission.
\end{abstract}

DOI: 10.1103/PhysRevA.83.053421

PACS number(s): $33.80 . \mathrm{Rv}, 33.70 .-\mathrm{w}, 42.50 . \mathrm{Hz}$

\section{INTRODUCTION}

In the last few decades, the availability of intense laser fields led to the observation of new phenomena which are nonlinear in the laser intensity. Among these, in high-order harmonic generation (HHG) processes the spectrum of the radiation emitted by atoms or molecules is characterized by a wide plateau of lines whose frequency is a multiple of the incident laser frequency $\omega_{L}$. One of the first attempts to understand the HHG mechanism was made by Corkum [1] by means of the three-step model. This model was the starting point for subsequent nonperturbative theories developed mainly within the framework of the strong-field approximation (SFA) (see [2] and references therein).

Several recent experiments have shown the potential of HHG as a spectroscopic tool for probing the orbital structure of molecules [3,4] and their nuclear dynamics [5-8], opening the route to combining attosecond temporal and sub-angstrom spatial information. In general, the main complication in dealing with laser-molecule interaction is in coping with the nuclear degrees of freedom, which are sometimes neglected by describing the nuclei as fixed point-like particles, an approximation justified by the fact that the motion of the nuclei is sufficiently slow with respect to the electron dynamics. This suggests that the state of the electrons adapts immediately to the changing distance of the nuclei and that the laser electric field has its most significant effect mainly on the electron dynamics. Although this is true if the laser pulse is short compared with the characteristic time scale of nuclear motion, for a long pulse the nuclear motion can become relevant and can induce significant modifications on the electron dynamics [3,6,9-18]. In particular, for a short few-cycle pulse [less than 10 optical cycles (o.c.) of a $800-n m$-wavelength laser pulse], it has been shown that the harmonics emitted by the heavier isotopes of $\mathrm{H}_{2}{ }^{+}$[9] and $\mathrm{H}_{2}$ [3,9] are more intense. This effect, confirmed in [6] for the $\mathrm{H}_{2}-\mathrm{D}_{2}$ molecular systems, is related to the fact that nuclear vibrations influence the contribution of a single electron trajectory to the HHG emission through the vibrational autocorrelation function defined in [3].

In this paper we show that the pulse length strongly affects the characteristics of the emitted radiation. In particular we find that in the long-pulse regime the activated dissociation process of the $\mathrm{H}_{2}{ }^{+}$molecular ion and of its isotopes can reverse the isotopic trend discussed in $[3,6,9]$.

\section{THE MODEL}

Our investigation has been performed by numerically solving the time-dependent Schrödinger equation (TDSE) for the electron and nuclear coordinates of hydrogen-like molecular ions driven by intense laser fields. Since in our calculations the laser field is linearly polarized along the internuclear axis, we use a one-dimensional approach to describe the time evolution of the system at issue. This assumption can be validated by considering that the ion has been preliminarily aligned with the laser field [19], although we are aware that for long-lasting pulses this assumption is questionable. In fact, for long pulses, nuclear rotational diffusion might destroy the alignment with the laser field. This effect might be taken into account by averaging our results over an ensemble of randomly oriented molecules. We shall not do this here in order to keep the mathematics as simple as possible. Moreover, the results of this work should be regarded as preliminary results of further investigations in which the molecular rotation is taken into account. Thus we solve the following TDSE:

$$
\begin{aligned}
i \hbar \frac{\partial}{\partial t} \psi(x, R, t)= & {\left[\left(-\frac{\hbar^{2}}{2 m} \frac{\partial^{2}}{\partial x^{2}}-\frac{\hbar^{2}}{2 \mu} \frac{\partial^{2}}{\partial R^{2}}\right)\right.} \\
& \left.+U(x, R)+V_{L}(x, t)\right] \psi(x, R, t),
\end{aligned}
$$

where $x$ and $R$ indicate, respectively, the electron and the internuclear distance coordinates, $m$ is the electron mass, and $\mu=\frac{M_{1} M_{2}}{M_{1}+M_{2}}$ is the reduced mass of the two nuclei (with $M_{1}$ and $M_{2}$ being the nuclear masses). $U(x, R)$ is the soft-core Coulomb interaction potential energy [20] with a screen soft-core parameter $A=a_{0}^{2}$ and $V_{L}(x, t)=\operatorname{ex} E(t)$ is the laser-electron interaction energy in the length gauge. Equation (1) is solved by means of a split-operator technique [21] using an electron spatial box $L_{x}=512 a_{0}$ wide with a numerical grid spacing $\Delta x=0.25 a_{0}$ and a nuclear spatial box $L_{R}=50 a_{0}$ wide with a numerical grid spacing $\Delta R=0.05 a_{0}$, with $a_{0}$ being the Bohr radius. For the laser field $E(t)$ we have adopted a sine-squared shaped profile with an intensity and a wavelength of $I_{0}=2 \times 10^{14} \mathrm{~W} / \mathrm{cm}^{2}$ and $\lambda=780 \mathrm{~nm}$, respectively. 

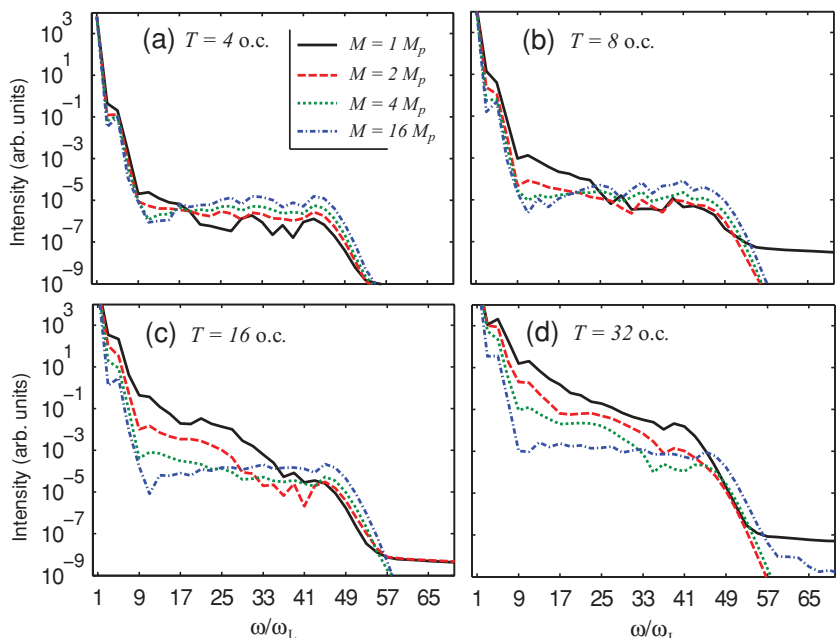

FIG. 1. (Color online) Envelopes of the intensity of the emitted spectra from the $\mathrm{H}_{2}{ }^{+}$-like molecular ions in the presence of an intense laser field $\left(I_{0}=2 \times 10^{14} \mathrm{~W} / \mathrm{cm}^{2}\right.$ and $\left.\lambda=780 \mathrm{~nm}\right)$ for different pulse durations. $M_{p}$ is the proton mass.

\section{RESULTS}

In Fig. 1 we show the envelopes of the radiation spectra emitted by different molecular isotopes $\left(\mathrm{H}_{2}{ }^{+}, \mathrm{D}_{2}{ }^{+}\right.$, and two artificial isotopes with nuclear mass $M_{1}=M_{2}=M=4 M_{p}$ and $\left.16 M_{p}\right)$ for different pulse durations $(4,8,16$, and 32 o.c.), where $M_{p}=1836.27 \mathrm{~m}$. It should be pointed out that we use the term artificial isotopes with a small abuse of language. Our aim is to study the effects of nuclear dynamics on the HHG spectrum. Thus when we consider an artificial isotope, note that this is just an artifact to better understand how the nuclear dynamics is involved in the mechanism of HHG and thus we do not refer to any real diatomic molecule. This figure, in addition to the typical HHG spectral shape, exhibits a peculiar dependence of the spectra on the duration of the laser pulse. In particular, it is evident that for short pulses (4 and 8 o.c.) the emitted harmonics are more intense for the heavier isotopes, while there is an opposite trend for longer pulses (the differences being about two orders of magnitude).

In order to give a quantitative explanation of this dependence, we write the solution of Eq. (1) in the form

$$
\psi(x, R, t)=\sum_{k} \chi_{k}(R, t) \Phi_{k}(x ; R),
$$

where the $\Phi_{k}(x ; R)$ are eigenfunctions corresponding to the free hamiltonian $H(R)$ of Eq. (1), with nuclei frozen at distance $R$, given by

$$
H(R)=-\frac{\hbar^{2}}{2 m} \frac{\partial^{2}}{\partial x^{2}}+U(x ; R),
$$

and whose eigenvalue equation is

$$
H(R) \Phi_{k}(x ; R)=\mathcal{E}_{k}(R) \Phi_{k}(x ; R),
$$

the $k$ index running over both bound and continuum states and $R$ being considered as a parameter. The $\chi_{k}(R, t)$ functions, formally expansion coefficients in (2), play the role of nuclear wave functions whose temporal evolution is related to the corresponding potential energy surfaces (PES) $\mathcal{E}_{k}(R)$ [see
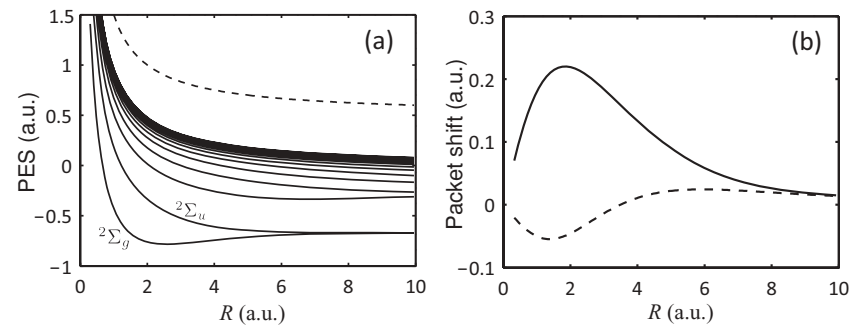

FIG. 2. (a) Numerically evaluated energy surfaces $\mathcal{E}_{k}(R)$ for the $\mathrm{H}_{2}{ }^{+}$-like molecular ions. The continuous lines represent the bound energy levels. The corresponding ${ }^{2} \Sigma_{g}$ and ${ }^{2} \Sigma_{u}$ states are marked; the dashed line is one of the energy levels in the continuum. (b) Relative shift accumulated by the nuclear wave packets $\chi_{k}(R, t)$ evolving on different energy surfaces. The continuous line represents the shift between the ground and $c$ level; the dashed line represents the shift between the first excited and $c$ level.

Fig. 2(a)] and is given, substituting Eq. (2) in (1), by solving the coupled equations

$$
\begin{aligned}
i \hbar \frac{\partial}{\partial t} \chi_{k}(R, t) \\
=\left[-\frac{\hbar^{2}}{2 \mu} \frac{\partial^{2}}{\partial R^{2}}+\mathcal{E}_{k}(R)\right] \chi_{k}(R, t) \\
\quad-E(t) \sum_{l} d_{k l}(R) \chi_{l}(R, t) \\
\quad+\sum_{l}\left[T_{k l}(R)+\frac{1}{\mu} P_{k l}(R)\left(-i \hbar \frac{\partial}{\partial R}\right)\right] \chi_{l}(R, t),
\end{aligned}
$$

where

$$
\begin{gathered}
d_{k l}(R)=\left\langle\Phi_{k}(x ; R)|-e x| \Phi_{l}(x ; R)\right\rangle, \\
T_{k l}(R)=\left\langle\Phi_{k}(x ; R)\left|-\frac{\hbar^{2}}{2 \mu} \frac{\partial^{2}}{\partial R^{2}}\right| \Phi_{l}(x ; R)\right\rangle, \\
P_{k l}(R)=\left\langle\Phi_{k}(x ; R)\left|-i \hbar \frac{\partial}{\partial R}\right| \Phi_{l}(x ; R)\right\rangle
\end{gathered}
$$

are the matrix elements of the electron dipole moment, nuclear kinetic term, and nuclear momentum, respectively (where in the bra-ket notation the integration is intended over the $x$ coordinate). Furthermore, it should be emphasized that Eq. (5) is given here in order to gain a better understanding of the physical meaning of the $\chi_{k}(R, t)$ functions. In this work we do not solve this equation explicitly; on the contrary, the reference to the $\chi_{k}(R, t)$ will be useful in what follows in order to appreciate the physical mechanism responsible for the obtained results.

The spectrum of the emitted radiation can be evaluated by means of the Fourier transform of the quantum averaged dipole moment of the molecule:

$D(\omega) \propto \int d t e^{-i \omega t} \sum_{k, l} \int d R d_{k l}(R) \chi_{k}(R, t) \chi_{l}^{*}(R, t)$.

In this expression $d_{k l}(R)$ varies more slowly with $R$ than the $\chi_{k}(R, t) \chi_{l}^{*}(R, t)$ term so that the former can be considered as a constant. It should be noted that we are neglecting here, for the sake of simplicity, charge resonant enhancement of ionization. This might tend to displace the nuclear wave packet from the equilibrium separation, spoiling somewhat the assumption of 
constant $d_{k l}(R)$. Nevertheless, expression (9) indicates that the overlap integral $\int d R \chi_{k}(R, t) \chi_{l}^{*}(R, t)$ between nuclear wave functions corresponding to different PES is relevant in determining the amplitude of the emitted radiation. Furthermore, differently from the assumption in [3] (negligible ground-state depletion), we assume that the depletion of the total population of the first two lower energetic states or, in molecular spectroscopic notation, of the bonding ${ }^{2} \Sigma_{g}$ and antibonding ${ }^{2} \Sigma_{u}$ states is negligible since in the regime of long pulses the probability of populating excited levels becomes significant [22,23]. Therefore in the sum appearing in Eq. (9) only the overlap between the first two nuclear wave functions $\left[\chi_{0}(R, t)\right.$ and $\chi_{1}(R, t)$, corresponding to ${ }^{2} \Sigma_{g}$ and ${ }^{2} \Sigma_{u}$, respectively] with all the others is relevant; i.e., Eq. (9) can be approximately written as

$$
\begin{aligned}
D(\omega) \propto & \int d t e^{-i \omega t}\left[\sum_{l=1}^{\infty} \int d R \chi_{0}(R, t) \chi_{l}^{*}(R, t)\right. \\
& \left.+\sum_{l=2}^{\infty} \int d R \chi_{1}(R, t) \chi_{l}^{*}(R, t)+\text { c.c. }\right],
\end{aligned}
$$

meaning that the main contribution to the harmonic emission arises from the electron escaping to the continuum from either the ${ }^{2} \Sigma_{g}$ or ${ }^{2} \Sigma_{u}$ state.

In molecules the energy levels depend upon the nuclear degrees of freedom [see Fig. 2(a)]. This means that the harmonic emission could depend on the region explored by the nuclear coordinates. For this reason, our interest is focused on estimating the contribution of each of the two terms in (10) when the nuclear wave packet moves around a given $R_{c}$.

To this end, we can assume that the nuclear wave packets are described, at any time $t$, by Gaussian wave packets with fixed spread. This assumption is justified if the molecule makes small oscillations around the equilibrium position so that, in this region, we can approximate the ground PES $\mathcal{E}_{0}(R)$ by a harmonic potential. In this case, the time evolution of the nuclear wave packet can be considered as that of a forced quantum harmonic oscillator which evolves on a coherent state [24].

Since we are interested in calculating the contribution to a given harmonic when the nuclear wave packet is centered at position $R_{c}$ at time $t_{c}$, the integral (10) must be evaluated on a characteristic time interval $\Delta \tau$ in which the process of harmonic emission occurs (less than the laser period so as presumed in the three-step model). Therefore the relation (10) becomes

$$
\begin{aligned}
D_{R_{c}}(\omega) \propto & \int_{t_{c}}^{t_{c}+\Delta \tau} d t e^{-i \omega t}\left[\sum_{l=1}^{\infty} \int d R \chi_{0}(R, t) \chi_{l}^{*}(R, t)\right. \\
& \left.+\sum_{l=2}^{\infty} \int d R \chi_{1}(R, t) \chi_{l}^{*}(R, t)+\text { c.c. }\right] .
\end{aligned}
$$

In order to estimate such integral, we take into account the vibrational autocorrelation function described in [3] and we modify the three-step model as follows:

(i) In the first step, at time $t_{1}=t_{c}$, the electron undergoes a transition from either the ground state $\Phi_{0}\left(x ; R_{c}\right)\left({ }^{2} \Sigma_{g}\right)$ or the first excited state $\Phi_{1}\left(x ; R_{c}\right)\left({ }^{2} \Sigma_{u}\right)$ to a Volkov state $\Phi_{k_{1}}^{V}\left(x, t_{1}\right)$. Due to celerity of the electronic transition and according to the sudden approximation [25], the nuclear wave function $\chi_{i}(R, t)$ ( $i=0,1$ ), appropriately weighted and centered at $R=R_{c}$, is transferred unaltered to the $k_{1}$ level. In other words, the electronic transition is weighted by the integral

$$
I_{1}=\int d R \chi_{i}\left(R, t_{1}\right) \chi_{k_{1}}^{*}\left(R, t_{1}\right), \quad i=0,1 .
$$

(ii) In the second step, under the influence of the laser field, the electron propagates in the interval from $t_{1}$ to $t_{2}=t_{c}+\Delta \tau$ from the $\Phi_{k_{1}}^{V}\left(x, t_{1}\right)$ to the $\Phi_{k_{2}}^{V}\left(x, t_{2}\right)$ state and the corresponding nuclear wave packet evolves from the $\chi_{k_{1}}\left(R, t_{1}\right)$ to the $\chi_{k_{2}}\left(R, t_{2}\right)$ state; this propagation, lasting a fraction of the laser period, implies that the $\chi_{k_{2}}\left(R, t_{2}\right)$ nuclear state at $t=t_{2}$ will be centered at a different position $R_{c^{\prime}}$.

(iii) In the third step, at time $t_{2}$, the electron recombines to either the ground bonding or the first excited antibonding state and the related harmonic emission is weighted by a factor given by the integral

$$
I_{2}=\int d R \chi_{k_{2}}\left(R, t_{2}\right) \chi_{i}^{*}\left(R, t_{2}\right), \quad i=0,1 .
$$

In addition, in the second step we assume that the shape of the $\chi_{k_{2}}\left(R, t_{2}\right)$ packet remains unchanged. This can be justified since in the time interval $\Delta \tau$ in which the process of harmonic emission occurs, the incremental spread of the nuclear wave packet can be considered negligible. Indeed, as reported in [24], the spread of a Gaussian wave packet at time $t$ is given by (in a.u.)

$$
\sigma_{R}(t)^{2}=\sigma_{R}^{2}\left(1+\frac{t^{2}}{4 \sigma_{R}^{4} M^{2}}\right),
$$

where $\sigma_{R}$ is the wave-packet spread at $t=0$. Therefore, substituting in (14) the values of $\sigma_{R}(0.24,0.20,0.17$, and 0.12 a.u.) found in our numerical calculations and considering one half of the laser period, we get $\sigma_{R}(t) / \sigma_{R} \simeq$ $1.10,1.06,1.03$, and 1.01, respectively, for the four molecular species (from lighter to heavier).

Furthermore, during the second step, since the $k_{1}$ and $k_{2}$ states are in the continuum, we can decouple the nuclear from the electronic dynamics and consider the nuclear coordinate evolving in the repulsive internuclear potential. In order to have an idea of the influence of $I_{2}$ on the emission of harmonics we consider that, after the time interval $\Delta \tau$ elapsed in the second step, the two nuclear wave packets $\chi_{i}(R, t)(i=0,1)$ and $\chi_{k_{2}}(R, t)$ have accumulated a displacement $\Delta R$ in their respective peaks. As a consequence, because of the overlap integral $I_{2}$, the corresponding harmonic emission is inhibited depending on the relative displacement $\Delta R / \sigma_{R}$. This also suggests that the value of the shift $\Delta R$ gives an estimate of the vibrational autocorrelation function introduced in [3] and consequently of the degree of inhibition of the harmonic emission.

We can thus estimate qualitatively the effects of $I_{2}$ by evaluating the shift $\Delta R$ which depends on the relative motion of the two wave packets moving on the respective energy surfaces. To this end, we can evaluate the dynamics of the nuclear wave packets in the bound and in the continuum levels by considering two classical particles, one of which moves on the potential surface $\mathcal{E}_{i}(R)(i=0,1)$ and the other on a surface $\mathcal{E}_{c}(R)$ (where subscript $c$ stands for continuum). This means 
that, after a time $\Delta \tau$, the displacement of the two particles will approximately be

$$
\Delta R \simeq \frac{1}{2} a_{\mathrm{rel}} \Delta \tau^{2}
$$

where

$$
a_{\text {rel }}=a_{c}-a_{i}=-\left.\frac{1}{M} \frac{d}{d R}\left[\mathcal{E}_{c}(R)-\mathcal{E}_{i}(R)\right]\right|_{R=R_{c}}, \quad i=0,1,
$$

is the relative acceleration of the two particles at the position $R=R_{c}$ defined above in the first step. Extending this argument to the two wave packets evolving on the potential surfaces previously considered, one is tempted to conclude that the shift will be more pronounced for lighter isotopes (smaller $M$ ) with the consequence that the emission is more intense for the heavier ones. More precisely we must say that this conclusion is correct if the electron transitions start from the ground ${ }^{2} \Sigma_{g}$ state and it is consistent with the results shown in Fig. 1 (and also with results in $[3,6,14,15])$ for the short-pulse regime (4 and 8 o.c.), in which the probability of populating excited electronic levels is small. In this case the main contribution to the harmonic emission is given by transitions starting from the ground bonding state. For longer pulses, however, the dynamics is characterized by an increase of both the internuclear distance and the spread of the nuclear wave packet (see Fig. 3 for 32 o.c. pulse length). This increase is larger and more rapid for lighter isotopic species, which will soon explore regions of $R$ in which the probability of populating excited electronic levels is greater and where, consequently, the right-hand side of (16) for $i=1$ essentially vanishes; it is also evident from Fig. 3(a) where the drift velocity observed for lighter species emphasizes that the dissociation process is active and from Fig. 4 where we plot the population peak of the first excited state ${ }^{2} \Sigma_{u}$ as a function of the internuclear distance $R$ (obtained by using a fixed nuclei model). More precisely, this figure shows the population peak trend with the corresponding internuclear coordinate excursion (double-sided arrows) for different isotopes. As we can see from the upper panel, for a few-cycle laser pulse the systems explore a region of $R$ in which the probability of populating the ${ }^{2} \Sigma_{u}$ state is low. In contrast, for a longer laser pulse (lower panel) a more pronounced internuclear excursion for the lighter isotopes with a consequent increase of the ${ }^{2} \Sigma_{u}$ population is evident. This means that lighter isotopes will spend a significant fraction of time in the first excited state where $a_{\text {rel }} \sim 0$, thus leading to a behavior different from the one observed in the case of the
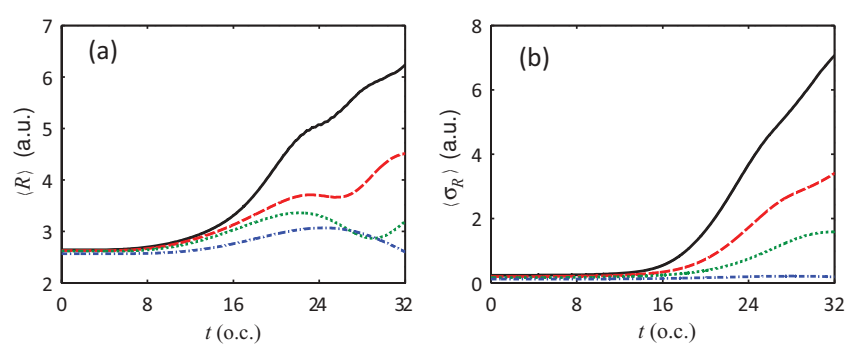

FIG. 3. (Color online) Temporal evolution of the mean values of (a) the internuclear distance $\langle R\rangle$ and (b) the correspondent spread $\left\langle\sigma_{R}\right\rangle$ for the 32 o.c. pulse length. The legend is as in Fig. 1 .
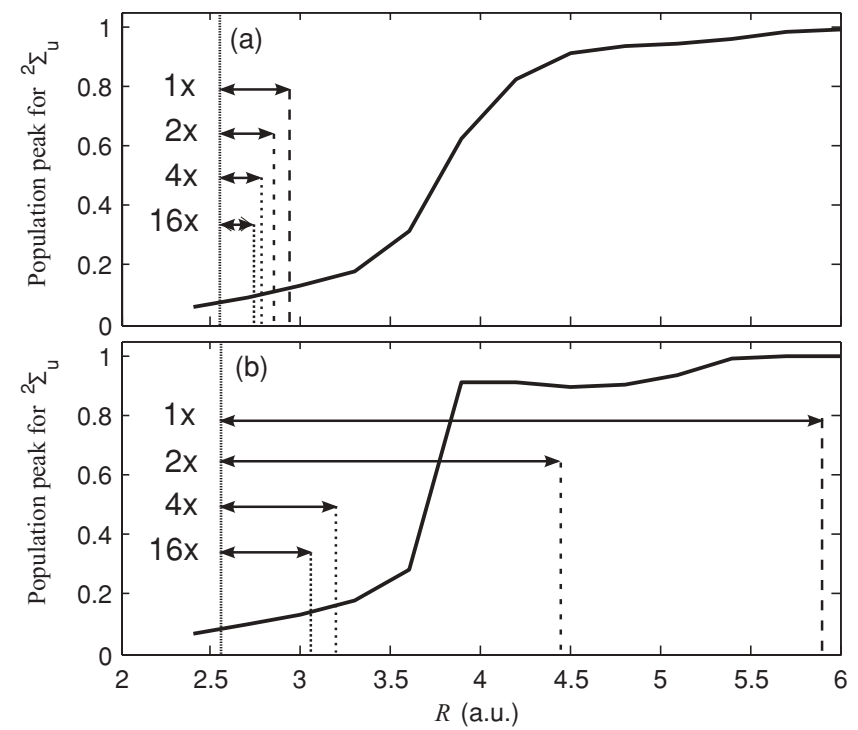

FIG. 4. Population peak of the ${ }^{2} \Sigma_{u}$ state as a function of the internuclear distance $R$ for (a) 4 o.c. and (b) 32 o.c. pulse length by considering a one-dimensional fixed nuclei model for the $\mathrm{H}_{2}{ }^{+}$ system. The double-sided arrows indicate the excursion of the mean value of the internuclear coordinate $\langle R\rangle$ obtained by solving Eq. (1) for the different isotopes. $1 \mathrm{x}$ corresponds to $\mathrm{H}_{2}{ }^{+}, 2 \mathrm{x}$ to $\mathrm{D}_{2}{ }^{+}$, and $4 \mathrm{x}$ and $16 x$ to the artificial isotopes with 4 and 16 times the $\mathrm{H}_{2}{ }^{+}$nuclear mass, respectively.

short-pulse regime: in fact a kind of reverse effect is observed in Fig. 1 for 16 and 32 o.c. pulse length.

Such a behavior can be understood from Fig. 2(a), where we observe that the slopes of the first excited and of a generic $c$ level in the continuum are almost the same in the region for $R \gtrsim 3$ a.u.. This means that the contribution to the harmonic emission is optimal when the electronic transition starts from the first excited level since $a_{\text {rel }} \sim 0$. To support this statement, we have calculated the relative acceleration $a_{\text {rel }}$ of two particles moving on the two pairs of energetic surfaces $\mathcal{E}_{0}(R), \mathcal{E}_{c}(R)$ and $\mathcal{E}_{1}(R), \mathcal{E}_{c}(R)$ and, consequently, by means of Eq. (15), the spatial shift $\Delta R$ between the two wave packets in half an o.c.. From the results, shown in Fig. 2(b) for the $\mathrm{H}_{2}{ }^{+}$ molecular ion (and for the other isotopes it suffices to scale the $y$ axis by the relative mass), we can see that the relative shift accumulated by the nuclear wave packets moving on the ground and $c$ levels is of the order of the nuclear wave-packet spread in the region around $R=2$ a.u., thus strongly inhibiting the harmonic emission because of the poor overlap of the wave packets. On the other hand, the shift of the wave packets moving on the first level and on a $c$ level approaches zero for $R \simeq 3$ a.u. and it is always smaller than the former shift. This suggests that lighter isotopes, having a greater chance to explore this region ( $R \geqslant 3$ a.u.), emit a more intense radiation, as confirmed by our results in Figs. 1(c) and 1(d). In other words, in the region in which $R \geqslant 3$ a.u. we observe an increase of the probability of populating the first excited antibonding state, which contributes significantly to the harmonic emission.

Finally, we briefly comment on the transportability of our results. It should be noted that we are aware that it is not simple to deal with $\mathrm{H}_{2}{ }^{+}$and $\mathrm{D}_{2}{ }^{+}$in a HHG experiment and we also believe that the results reported in this work probably do not 
hold for the corresponding molecular systems $\mathrm{H}_{2}$ and $\mathrm{D}_{2}$ due to their different spectroscopic properties. At this stage we do not have any suggestion about possible transportability to other molecular systems. However, we are confident that our results can provide a unified understanding of the response of a molecular system interacting with an intense laser field and we hope that they can suggest possible practical applications due to the considerable increase in the intensity of emitted radiation obtained in our simulations.

\section{CONCLUSIONS}

In summary, we have discussed how the nuclear dynamics affects the intensity of the emitted harmonics. More precisely, we observe a different behavior depending on the length of the pulse considered: for short pulses, as already obtained in $[3,6,14,15]$, heavier isotopes emit more intense highorder harmonic lines; in contrast, for long pulses an inverse phenomenon is observed, with the differences being about two order of magnitude. This can be used as an efficient tool for the enhancement of the harmonic emission and as a valuable spectroscopic tool.
The modification of the three-step model, in which we have assumed that the transitions starting from the ground bonding state and from the first excited antibonding state contribute to the harmonic emission, is able to explain the different behavior described by our numerical results for the two pulse regimes. In particular, we have shown that the inverse behavior in the long-pulse regime is due to the transitions starting from the antibonding state. In other words, the activation of the molecular dissociation channel is responsible for the emission enhancement.

\section{ACKNOWLEDGMENTS}

We thank M. Ivanov and J. Marangos for useful discussions. This work makes use of results produced by the PI2S2 Project managed by the Consorzio COMETA, a project co-funded by the Italian Ministry of University and Research (MIUR) within the Piano Operativo Nazionale "Ricerca Scientifica, Sviluppo Tecnologico, Alta Formazione" (PON 2000-2006). More information is available at http://www.pi2s2.it and http://www.consorzio-cometa.it.
[1] P. B. Corkum, Phys. Rev. Lett. 71, 1994 (1993).

[2] M. Protopapas, C. H. Keitel, and P. L. Knight, Rep. Prog. Phys. 60, 389 (1997).

[3] M. Lein, Phys. Rev. Lett. 94, 053004 (2005).

[4] J. Itatani, J. Levesque, D. Zeidler, H. Niikura, H. Pépin, J. C. Kieffer, P. B. Corkum, and D. M. Villeneuve, Nature (London) 432, 867 (2004).

[5] T. Kanai, S. Minemoto, and H. Sakai, Nature (London) 435, 470 (2005).

[6] S. Baker, J. S. Robinson, C. A. Haworth, H. Teng, R. A. Smith, C. C. Chirilă, M. Lein, J. W. G. Tisch, and J. P. Marangos, Science 312, 424 (2006).

[7] S. Baker et al., Phys. Rev. Lett. 101, 053901 (2008).

[8] W. Li, X. Zhou, R. Lock, S. Patchkovskii, A. Stolow, H. C. Kapteyn, and M. M. Murnane, Science 322, 1207 (2008).

[9] C. C. Chirilă and M. Lein, J. Phys. B 39, S437 (2006).

[10] I. A. Gonoskov, M. Yu. Ryabikin, and A. M. Sergeev, J. Phys. B 39, S445 (2006).

[11] P. P. Corso, E. Fiordilino, and F. Persico, J. Phys. B 40, 1383 (2007).

[12] A. D. Bandrauk, S. Chelkowski, S. Kawai, and H. Lu, Phys. Rev. Lett. 101, 153901 (2008).

[13] J. Zhao and Z. Zhao, Phys. Rev. A 78, 053414 (2008).
[14] S. Patchkovskii, Phys. Rev. Lett. 102, 253602 (2009).

[15] A. D. Bandrauk, S. Chelkowski, and H. Lu, J. Phys. B 42, 075602 (2009).

[16] O. Smirnova, Y. Mairesse, S. Patchkovskii, N. Dudovich, D. Villeneuve, P. Corkum, and M. Yu. Ivanov, Nature (London) 460, 972 (2009).

[17] C. B. Madsen, M. Abu-samha, and L. B. Madsen, Phys. Rev. A 81, 043413 (2010).

[18] M. Falge, V. Engel, and M. Lein, Phys. Rev. A 81, 023412 (2010).

[19] H. Stapelfeldt, Rev. Mod. Phys. 75, 543 (2003).

[20] J. Javanainen, J. H. Eberly, and Q. Su, Phys. Rev. A 38, 3430 (1988).

[21] M. D. Feit and A. Steiger, J. Comput. Phys. 47, 412 (1982).

[22] T. Zuo, S. Chelkowski, and A. D. Bandrauk, Phys. Rev. A 48, 3837 (1993).

[23] T. Seideman, M. Y. Ivanov, and P. B. Corkum, Phys. Rev. Lett. 75, 2819 (1995).

[24] E. Merzbacher, Quantum Mechanics, 2nd ed. (Wiley, New York, 1970).

[25] L. I. Schiff, Quantum Mechanics (McGraw-Hill, New York, 1968). 